\title{
Robust stability analysis for BAM neural networks of neutral type with time-varying delays and linear fractional uncertainties
}

\author{
Yunxi Zhang ${ }^{1,}$ a , Jia Liu ${ }^{1, b}$, Yongxin $\mathrm{Li}^{2}$ \\ ${ }^{1}$ Tianjin Key Laboratory of Information Sensing and Intelligent Control, School of Automation and \\ Electrical Engineering, Tianjin University of Technology and Education, Tianjin 300222., China \\ ${ }^{2}$ College of Computer and Control Engineering, Nankai University, Tianjin 300071, China \\ aemail: zyun2004@126.com, bemail: liujia_0704@163.com
}

Keywords: Bidirectional associative memory neural networks; Robust stability; Time-varying delays; Linear fractional form; Lyapunov-Krasovskii functional

\begin{abstract}
This paper investigates the problem of robust stability for bidirectional associative memory (BAM) neural networks of neutral type with time-varying delays and linear fractional uncertainties. By employing integral equality and constructing a new Lyapunov-Krasovskii functional, a sufficient criterion is proposed on robust asymptotic stability for a given BAM neural networks with linear fractional uncertainties. The parameters uncertainties are expressed in a linear fractional form, which includes the norm bounded uncertainties as a special case. Numerical examples are provided to illustrate the effectiveness and less conservatism of the main result.
\end{abstract}

\section{Introduction}

Over the past decades, bidirectional associative memory (BAM) neural networks have received considerable attention due to their extensive applications in the fields of pattern recognition, artificial intelligence, automatic control engineering because of its better abilities of information memory and information association. Recently, a great number of researchers have studied stability properties of the neural networks and presented various sufficient conditions for the asymptotic or exponential stability of the BAM neural networks, see, e.g. [1-3].

Usually, the integration and communication delays are unavoidably encountered both in biological and artificial neural systems, which frequently leads to an oscillation, divergence and furthermore, or instability of neural networks. So, some results on stability of BAM networks with time delays have been developed in [1,2]. In practice, the stability of a neural network may often be destroyed by its unavoidable uncertainties due to the existence of modeling errors, external disturbance and parameter fluctuation in the applications and designs of neural networks. Therefore, when both the time delays and parameter uncertainties are taken into account in neural networks, robust stability conditions have been given in [4,5].

In addition, owing to the complicated dynamic properties of the neural cells in the real world, the existing neural network models in many cases can not characterize the properties of a neural reaction process precisely. It is natural and important that systems will contain some information about the derivative of the past state to further describe and model the dynamics for such complex neural reactions. This new type of neural networks is called neutral neural networks or neural networks of neutral-type. However, up to date, the stability analysis for neural networks of neutral-type has been rarely investigated [6,7].

In this paper, we focus on the problem of the robust asymptotic stability for BAM neural networks of neutral type with time-varying delays and linear fractional uncertainties. By introducing a new integral equality and constructing a Lyapunov-Krasovskii functional, novel delay-dependent condition is established checking the asymptotic stability for the considered BAM neural system. The parameters uncertainties are expressed in a linear fractional form, which includes the norm bounded uncertainties as a special case. Finally, a numerical example is provided to illustrate the effectiveness of the proposed result, which is less conservative than that in [6,7]. 


\section{Problem Statement and Preliminaries}

Consider the following uncertain delayed BAM neural network of neutral-type:

$$
\begin{aligned}
& \dot{u}(t)=-A(t) u(t)+W_{1}^{T}(t) \tilde{f}(v(t-\tau(t)))+W_{2}(t) \dot{u}(t-h)+\omega, \\
& \dot{v}(t)=-B(t) v(t)+V_{1}^{T}(t) \tilde{g}(u(t-\sigma(t)))+V_{2}(t) \dot{v}(t-d)+v,
\end{aligned}
$$

where $u(t)=\left[u_{1}(t), u_{2}(t), \cdots, u_{n}(t)\right]^{T} \in \mathcal{R}^{n}$ and $v(t)=\left[v_{1}(t), v_{2}(t), \cdots, v_{m}(t)\right]^{T} \in \mathcal{R}^{m}$ are the neuron state vectors, $\tilde{f}(\cdot)=\left[\tilde{f}_{1}(\cdot), \tilde{f}_{2}(\cdot), \cdots, \tilde{f}_{m}(\cdot)\right]$ and $\tilde{g}(\cdot)=\left[\tilde{g}_{1}(\cdot), \tilde{g}_{2}(\cdot), \cdots, \tilde{g}_{n}(\cdot)\right]$ are the activation functions, $\omega$ and $v$ denote the constant external inputs, $h>0$ and $d>0$ are neutral delays, $\tau(t)$ and $\sigma(t)$ are state time-varying delays, which are differentiable functions that satisfy

$$
0 \leq \tau(t) \leq \tau, \dot{\tau}(t) \leq \tau_{d}, 0 \leq \sigma(t) \leq \sigma, \dot{\sigma}(t) \leq \sigma_{d},
$$

for all $t>0$ and prescribed scalars $\tau \geq 0, \sigma \geq 0, \tau_{d} \geq 0, \sigma_{d} \geq 0 . A(t), W_{1}^{T}(t), W_{2}(t), B(t), V_{1}^{T}(t), V_{2}(t)$ are time-varying matrices which are assumed to be of the following form:

$$
\begin{aligned}
& {\left[\begin{array}{lll}
A(t) & W_{1}^{T}(t) & W_{2}(t) \\
B(t) & V_{1}^{T}(t) & V_{2}(t)
\end{array}\right]=\left[\begin{array}{ccc}
A & W_{1}^{T} & W_{2} \\
B & V_{1}^{T} & V_{2}
\end{array}\right]+\left[\begin{array}{l}
H_{1} \\
H_{2}
\end{array}\right] \Delta(t)\left[\begin{array}{lll}
Z_{1} & Z_{2} & Z_{3}
\end{array}\right],} \\
& \Delta(t)=F(t)[I-J F(t)]^{-1}, \quad 0<I-J^{T} J,
\end{aligned}
$$

where $A=\operatorname{diag}\left(a_{1}, a_{2}, \cdots, a_{n}\right)>0$ and $B=\operatorname{diag}\left(b_{1}, b_{2}, \cdots, b_{m}\right)>0$ are positive diagonal matrices, $W=\left(w_{i j}\right)_{m \times n}, V=\left(v_{i j}\right)_{n \times m}$ are interconnection matrices representing the weight coefficients of the neurons, $H_{1}, H_{2}, Z_{1}, Z_{2}, Z_{3}$ are known real constant matrices with appropriate dimensions and $F(t)$ is an unknown time-varying matrix function satisfying

$F^{T}(t) F(t) \leq I, \quad \forall t>0$.

Remark 1. It is worth noting that linear fractional uncertainties are more general than the usual norm-bounded uncertainties, because when $J=0$, the linear fractional uncertainties can be changed into the norm-bounded uncertainties.

In addition, we will assume the activation functions $\tilde{f}_{j}(\cdot)$ and $\tilde{g}_{i}(\cdot)$ in $(1)$ are satisfies the following conditions:

(A1): $\tilde{f}_{j}(\cdot)$ and $\tilde{g}_{i}(\cdot)$ are bounded on $\mathcal{R}, i=1,2, \cdots, n, j=1,2, \cdots, m$.

(A2): $\tilde{f}_{j}(\cdot)$ and $\tilde{g}_{i}(\cdot)$ are Lipschitz continuous, there exist real scalars $l_{j}>0, k_{i}>0$, such that $\left|\tilde{f}_{j}(x)-\tilde{f}_{j}(y)\right| \leq l_{j}|x-y|, \quad\left|\tilde{g}_{i}(x)-\tilde{g}_{i}(y)\right| \leq k_{i}|x-y|$, for any $x, y \in \mathcal{R}, i=1,2, \cdots, n, j=1,2, \cdots, m$.

By using the well-known Brouwer fixed point theorem, one can see that there exists one equilibrium point for system (1). Then system (1) can be written as

$\dot{x}(t)=-A(t) x(t)+W_{1}^{T}(t) f(y(t-\tau(t)))+W_{2}(t) \dot{x}(t-h)$,

$\dot{y}(t)=-B(t) y(t)+V_{1}^{T}(t) g(x(t-\sigma(t)))+V_{2}(t) \dot{y}(t-d)$.

From (A1) and (A2), we can derive that the activation functions $f_{j}(\cdot)$ and $g_{i}(\cdot)$ satisfy

(H1): $f_{j}(\cdot)$ and $g_{i}(\cdot)$ are bounded on $\mathcal{R}, i=1,2, \cdots, n, j=1,2, \cdots, m$.

$(\mathrm{H} 2): f_{j}(\cdot)$ and $g_{i}(\cdot)$ are Lipschitz continuous, there exist real scalars $l_{j}>0, k_{i}>0$, such that

$$
\left|f_{j}(x)-f_{j}(y)\right| \leq l_{j}|x-y|, \quad\left|g_{i}(x)-g_{i}(y)\right| \leq k_{i}|x-y|,
$$

for any $x, y \in \mathcal{R}, i=1,2, \cdots, n, j=1,2, \cdots, m$.

(H3): $f_{j}(0)=0, g_{i}(0)=0, i=1,2, \cdots, n, j=1,2, \cdots, m$.

\section{Main results}

In this section, we will present the delay-dependent conditions ensuring the robust asymptotic stability of the equilibrium point for delayed BAM neural network of neutral-type with linear fractional uncertainties.

Firstly, we consider the asymptotic stability criterion for the nominal system: 


$$
\begin{aligned}
& \dot{x}(t)=-A x(t)+W_{1}^{T} f(y(t-\tau(t)))+W_{2} \dot{x}(t-h), \\
& \dot{y}(t)=-B y(t)+V_{1}^{T} g(x(t-\sigma(t)))+V_{2} \dot{y}(t-d) .
\end{aligned}
$$

Theorem1. Under $(\mathrm{H} 1)-(\mathrm{H} 3)$, the origin of the delayed BAM neural network in (6) is asymptotically stable if there exist matrices $P=\left[\begin{array}{cc}P_{11} & P_{12} \\ * & P_{22}\end{array}\right]>0, Q=\left[\begin{array}{cc}Q_{11} & Q_{12} \\ * & Q_{22}\end{array}\right]>0, R_{1}>0, R_{2}>0, R_{3}>0$, $R_{4}>0, S_{1}>0, S_{2}>0, T=\left[\begin{array}{cc}T_{11} & T_{12} \\ * & T_{22}\end{array}\right]>0, U=\left[\begin{array}{cc}U_{11} & U_{12} \\ * & U_{22}\end{array}\right]>0$, diagonal matrices $Y_{1}>0, Y_{2}>0$ and matrices $M_{i}, N_{i}(i=1, \cdots, 6), \bar{M}_{j}, \bar{N}_{j}(j=1,2,3)$ with appropriate dimensions respectively, such that the following inequality holds:

$$
\Theta=\left[\begin{array}{ccc}
\Psi & \sigma \Gamma_{1}^{T} & \tau \Gamma_{2}^{T} \\
* & -\sigma S_{1} & 0 \\
* & * & -\tau S_{2}
\end{array}\right]<0,
$$

where $\Psi=\Psi^{T}=\left(\Psi_{i j}\right)_{12 \times 12}$, with

$$
\begin{aligned}
& \Psi_{11}=R_{1}+T_{11}+M_{1}+M_{1}^{T}-\bar{M}_{1} A-A^{T} \bar{M}_{1}^{T}+K R_{3} K, \Psi_{12}=P_{11}+T_{12}^{T}-\bar{M}_{1}-A^{T} \bar{M}_{2}^{T}+M_{2}, \Psi_{13}=-M_{1}+M_{3}^{T}, \\
& \Psi_{14}=M_{4}^{T}, \Psi_{15}=P_{12}-A^{T} \bar{M}_{3}^{T}+\bar{M}_{1} W_{2}^{T}+M_{5}^{T}, \Psi_{16}=M_{6}^{T} \Psi_{17}=\cdots=\Psi_{1,11}=0, \Psi_{1,12}=\bar{M}_{1} W_{1}^{T}, \\
& \Psi_{22}=T_{22}+\sigma S_{1}-\bar{M}_{2}-\bar{M}_{2}^{T}, \Psi_{23}=-M_{2}, \Psi_{24}=P_{12}, \Psi_{25}=\bar{M}_{2} W_{2}-\bar{M}_{3}^{T}, \Psi_{26}=\cdots=\Psi_{2,11}=0, \Psi_{2,12}=\bar{M}_{2} W_{1}^{T} \text {, } \\
& \Psi_{33}=-\left(1-\sigma_{d}\right) R_{1}-M_{3}-M_{3}^{T}+K Y_{1} K, \Psi_{34}=-M_{4}^{T}, \Psi_{35}=-M_{5}^{T}, \Psi_{36}=-M_{6}^{T}, \Psi_{37}=\cdots=\Psi_{3,12}=0 \text {, } \\
& \Psi_{44}=-T_{11}, \Psi_{45}=P_{22}-T_{22}, \Psi_{46}=\cdots=\Psi_{4,12}=0 \text {, } \\
& \Psi_{55}=-T_{22}+\bar{M}_{3} W_{2}+W_{2}^{T} \bar{M}_{3}^{T}, \Psi_{56}=\cdots=\Psi_{5,11}=0, \Psi_{5,12}=M_{3} W_{1}^{T}, \Psi_{66}=-\left(1-\sigma_{d}\right) R_{3}-Y_{1}, \Psi_{67}=V_{1} \bar{N}_{1}^{T}, \\
& \Psi_{68}=V_{1} \bar{N}_{2}^{T}, \Psi_{69}=0, \Psi_{6,10}=0, \Psi_{6,11}=V_{1} \bar{N}_{3}^{T}, \Psi_{6,12}=0, \Psi_{77}=R_{2}+U_{11}+N_{1}+N_{1}^{T}-\bar{N}_{1} B-B^{T} \bar{N}_{1}^{T}+L R_{4} L, \\
& \Psi_{78}=Q_{11}+U_{12}^{T}-\bar{N}_{1}-B^{T} \bar{N}_{2}^{T}+N_{2}, \Psi_{79}=-N_{1}+N_{3}^{T}, \Psi_{7,10}=N_{4}^{T}, \Psi_{7,11}=Q_{12}-B^{T} \bar{N}_{3}^{T}+\bar{N}_{1} V_{2}^{T}+N_{5}^{T}, \Psi_{7,12}=N_{6}^{T} \text {, } \\
& \Psi_{88}=U_{22}+\tau S_{2}-\bar{N}_{2}-\bar{N}_{2}^{T}, \Psi_{89}=-N_{2}, \Psi_{8,10}=Q_{12}, \Psi_{8,11}=\bar{N}_{2} V_{2}-\bar{N}_{3}^{T}, \Psi_{8,12}=0 \text {, } \\
& \Psi_{99}=-\left(1-\tau_{d}\right) R_{2}-N_{3}-N_{3}^{T}+L Y_{2} L, \Psi_{9,10}=-N_{4}^{T}, \Psi_{9,11}=-N_{5}^{T}, \Psi_{9,12}=-N_{6}^{T} \text {, } \\
& \Psi_{10,10}=-U_{11}, \Psi_{10,11}=Q_{22}-U_{12}, \Psi_{10,12}=0, \Psi_{11,11}=-U_{22}+\bar{N}_{3} V_{2}+V_{2}^{T} \bar{N}_{3}^{T}, \Psi_{11,12}=0, \Psi_{12,12}=-\left(1-\tau_{d}\right) R_{4}-Y_{2} \text {, } \\
& L=\operatorname{diag}\left(l_{1}, l_{2}, \cdots, l_{m}\right)>0, \quad K=\operatorname{diag}\left(k_{1}, k_{2}, \cdots, k_{n}\right)>0 \text {, } \\
& \Gamma_{1}=\left[\begin{array}{lllllllll}
M_{1} & M_{2} & M_{3} & M_{4} & M_{5} & M_{6} & 0 & \cdots & 0
\end{array}\right]_{1 \times 12}, \Gamma_{2}=\left[\begin{array}{lllllllll}
0 & \cdots & 0 & N_{1} & N_{2} & N_{3} & N_{4} & N_{5} & N_{6}
\end{array}\right]_{1 \times 12} \text {. }
\end{aligned}
$$

Proof. Develop the following Lyapunov-Krasovskii functional candidate as

$$
V\left(x_{t}, y_{t}\right)=\sum_{i=1}^{4} V_{i}\left(x_{t}, y_{t}\right) \text {, }
$$

where

$$
\begin{aligned}
& V_{1}\left(x_{t}, y_{t}\right)=\left[\begin{array}{ll}
x^{T}(t) & x^{T}(t-h)
\end{array}\right] P\left[\begin{array}{c}
x(t) \\
x(t-h)
\end{array}\right]+\left[\begin{array}{ll}
y^{T}(t) & y^{T}(t-d)
\end{array}\right] Q\left[\begin{array}{c}
y(t) \\
y(t-d)
\end{array}\right], \\
& V_{2}\left(x_{t}, y_{t}\right)=\int_{t-\sigma(t)}^{t} x^{T}(s) R_{1} x(s) d s+\int_{t-\tau(t)}^{t} y^{T}(s) R_{2} y(s) d s \\
& +\int_{t-\sigma(t)}^{t} g^{T}(x(s)) R_{3} g(x(s)) d s+\int_{t-\tau(t)}^{t} f^{T}(y(s)) R_{4} f(y(s)) d s, \\
& V_{3}\left(x_{t}, y_{t}\right)=\int_{-\sigma}^{0} \int_{t+\beta}^{t} \dot{x}^{T}(\alpha) S_{1} \dot{x}(\alpha) d \alpha d \beta+\int_{-\tau}^{0} \int_{t+\beta}^{t} \dot{y}^{T}(\alpha) S_{2} \dot{y}(\alpha) d \alpha d \beta, \\
& V_{4}\left(x_{t}, y_{t}\right)=\int_{t-h}^{t}\left[\begin{array}{ll}
x^{T}(s) & \dot{x}^{T}(s)
\end{array}\right] T\left[\begin{array}{c}
x(s) \\
\dot{x}(s)
\end{array}\right] d s+\int_{t-d}^{t}\left[\begin{array}{ll}
y^{T}(s) & \dot{y}^{T}(s)
\end{array}\right] U\left[\begin{array}{c}
y(s) \\
\dot{y}(s)
\end{array}\right] d s .
\end{aligned}
$$

Calculating the time derivative of $V\left(x_{t}, y_{t}\right)$ along the trajectory of system (6) gives 


$$
\begin{aligned}
& \dot{V}_{1}\left(x_{t}, y_{t}\right)=2\left[x^{T}(t) \quad x^{T}(t-h)\right]\left[\begin{array}{cc}
P_{11} & P_{12} \\
* & P_{22}
\end{array}\right]\left[\begin{array}{c}
\dot{x}(t) \\
\dot{x}(t-h)
\end{array}\right]+2\left[\begin{array}{ll}
y^{T}(t) & y^{T}(t-d)
\end{array}\right]\left[\begin{array}{cc}
Q_{11} & Q_{12} \\
* & Q_{22}
\end{array}\right]\left[\begin{array}{c}
\dot{y}(t) \\
\dot{y}(t-d)
\end{array}\right], \\
& \dot{V}_{2}\left(x_{t}, y_{t}\right) \leq x^{T}(t) R_{1} x(t)-\left(1-\sigma_{d}\right) x^{T}(t-\sigma(t)) R_{1} x(t-\sigma(t))+y^{T}(t) R_{2} y(t) \\
& -\left(1-\tau_{d}\right) y^{T}(t-\tau(t)) R_{2} y(t-\tau(t))+x^{T}(t) K R_{3} K x(t)-\left(1-\sigma_{d}\right) g^{T}(x(t-\sigma(t))) R_{3} g(x(t-\sigma(t))) \\
& +y^{T}(t) L R_{4} L y(t)-\left(1-\tau_{d}\right) f^{T}(y(t-\tau(t))) R_{4} f(y(t-\tau(t))) \text {, } \\
& \dot{V}_{3}\left(x_{t}, y_{t}\right) \leq \sigma \dot{x}^{T}(t) S_{1} \dot{x}(t)+2 \xi_{1}^{T}(t) M[x(t)-x(t-\sigma(t))]+\sigma \xi_{1}^{T}(t) X_{1} \xi_{1}(t) \\
& -\int_{t-\sigma(t)}^{t}\left[\begin{array}{ll}
\xi_{1}^{T}(t) & \dot{x}^{T}(\alpha)
\end{array}\right]\left[\begin{array}{cc}
X_{1} & M \\
M^{T} & S_{1}
\end{array}\right]\left[\begin{array}{c}
\xi_{1}(t) \\
\dot{x}(\alpha)
\end{array}\right] d \alpha+\tau \dot{y}^{T}(t) S_{2} \dot{y}(t)+2 \xi_{2}^{T}(t) N[y(t)-y(t-\tau(t))] \\
& +\tau \xi_{2}^{T}(t) X_{2} \xi_{2}(t)-\int_{t-\tau(t)}^{t}\left[\xi_{2}^{T}(t) \quad \dot{y}^{T}(\alpha)\right]\left[\begin{array}{cc}
X_{2} & N \\
N^{T} & S_{2}
\end{array}\right]\left[\begin{array}{c}
\xi_{2}(t) \\
\dot{y}(\alpha)
\end{array}\right] d \alpha \\
& \dot{V}_{4}\left(x_{t}, y_{t}\right)=\left[\begin{array}{ll}
x^{T}(t) & \dot{x}^{T}(t)
\end{array}\right]\left[\begin{array}{cc}
T_{11} & T_{12} \\
* & T_{22}
\end{array}\right]\left[\begin{array}{c}
x(t) \\
\dot{x}(t)
\end{array}\right]-\left[\begin{array}{ll}
x^{T}(t-h) & \dot{x}^{T}(t-h)
\end{array}\right]\left[\begin{array}{cc}
T_{11} & T_{12} \\
* & T_{22}
\end{array}\right]\left[\begin{array}{l}
x(t-h) \\
\dot{x}(t-h)
\end{array}\right] \\
& +\left[\begin{array}{ll}
y^{T}(t) & \dot{y}^{T}(t)
\end{array}\right]\left[\begin{array}{cc}
U_{11} & U_{12} \\
* & U_{22}
\end{array}\right]\left[\begin{array}{c}
y(t) \\
\dot{y}(t)
\end{array}\right]-\left[\begin{array}{ll}
y^{T}(t-d) & \dot{y}^{T}(t-d)
\end{array}\right]\left[\begin{array}{cc}
U_{11} & U_{12} \\
* & U_{22}
\end{array}\right]\left[\begin{array}{c}
y(t-d) \\
\dot{y}(t-d)
\end{array}\right] .
\end{aligned}
$$

where

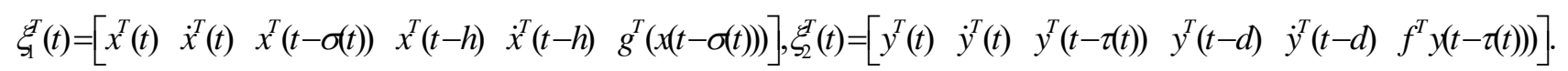

Observe that

$$
\begin{aligned}
& 0=2\left[\begin{array}{lll}
x^{T}(t) & \dot{x}^{T}(t) & \dot{x}^{T}(t-h)
\end{array}\right]\left[\begin{array}{l}
\bar{M}_{1} \\
\bar{M}_{2} \\
\bar{M}_{3}
\end{array}\right]\left[-\dot{x}(t)-A x(t)+W_{1}^{T} f(y(t-\tau(t)))+W_{2} \dot{x}(t-h)\right], \\
& 0=2\left[\begin{array}{lll}
y^{T}(t) & \dot{y}^{T}(t) & \dot{y}^{T}(t-d)
\end{array}\right]\left[\begin{array}{l}
\bar{N}_{1} \\
\bar{N}_{2} \\
\bar{N}_{3}
\end{array}\right]\left[-\dot{y}(t)-B y(t)+V_{1}^{T} g(x(t-\sigma(t)))+V_{2} \dot{y}(t-d)\right] .
\end{aligned}
$$

In light of (H1)-(H3), for diagonal matrices $Y_{1}>0, Y_{2}>0$, we can obtain

$$
\begin{aligned}
& 0 \leq x^{T}(t-\sigma(t)) K T_{1} K x(t-\sigma(t))-g^{T}(x(t-\sigma(t))) T_{1} g(x(t-\sigma(t))), \\
& 0 \leq y^{T}(t-\tau(t)) L T_{2} L y(t-\tau(t))-f^{T}(y(t-\tau(t))) T_{2} f(y(t-\tau(t))) .
\end{aligned}
$$

According to (9)-(11), we have

$$
\begin{aligned}
\dot{V}\left(x_{t}, y_{t}\right) \leq & \xi^{T}(t) \Psi \xi(t)+\sigma \xi_{1}^{T}(t) X_{1} \xi_{1}(t)-\int_{t-\sigma(t)}^{t}\left[\begin{array}{cc}
\xi_{1}^{T}(t) & \dot{x}^{T}(\alpha)
\end{array}\right]\left[\begin{array}{cc}
X_{1} & M \\
M^{T} & S_{1}
\end{array}\right]\left[\begin{array}{c}
\xi_{1}(t) \\
\dot{x}(\alpha)
\end{array}\right] d \alpha \\
& +\tau \xi_{2}^{T}(t) X_{2} \xi_{2}(t)-\int_{t-\tau(t)}^{t}\left[\begin{array}{cc}
\xi_{2}^{T}(t) & \dot{y}^{T}(\alpha)
\end{array}\right]\left[\begin{array}{cc}
X_{2} & N \\
N^{T} & S_{2}
\end{array}\right]\left[\begin{array}{c}
\xi_{2}(t) \\
\dot{y}(\alpha)
\end{array}\right] d \alpha .
\end{aligned}
$$

Let $X_{1}=M S_{1}^{-1} M^{T}, X_{2}=N S_{2}^{-1} N^{T}$, one can see that $\left[\begin{array}{cc}X_{1} & M \\ M^{T} & S_{1}\end{array}\right] \geq 0,\left[\begin{array}{cc}X_{2} & N \\ N^{T} & S_{2}\end{array}\right] \geq 0$.

Then, we have $\dot{V}\left(x_{t}, y_{t}\right) \leq \xi^{T}(t)\left[\Psi+\sigma \Gamma_{1} S_{1}^{-1} \Gamma_{1}^{T}+\tau \Gamma_{2} S_{2}^{-1} \Gamma_{2}^{T}\right] \xi(t)$.

By Schur's complements lemma, we know $\Psi+\sigma_{1} S_{1}^{-1} \Gamma_{1}^{T}+\tau \Gamma_{2} S_{2}^{-1} \Gamma_{2}^{T}<0$, which is equivalent to $\Theta<0$ in (7). Therefore, we have $\dot{V}\left(x_{t}, y_{t}\right) \leq 0$. Then, by the standard Lyapunov functional theory, it concludes that the origin of the given delayed BAM neural network system (6) is asymptotically stable. This completes the proof.

Next, we consider the robust asymptotic stability for the given BAM neural networks (5).

Theorem 2. Under (H1)-(H3), the origin of the delayed BAM neural network in (5) is robust asymptotically stable if there exist scalars $\epsilon_{1}>0, \epsilon_{2}>0$, matrices $P=\left[\begin{array}{cc}P_{11} & P_{12} \\ * & P_{22}\end{array}\right]>0, Q=\left[\begin{array}{cc}Q_{11} & Q_{12} \\ * & Q_{22}\end{array}\right]>0$, 
$R_{1}>0, R_{2}>0, R_{3}>0, R_{4}>0, S_{1}>0, S_{2}>0, T=\left[\begin{array}{cc}T_{11} & T_{12} \\ * & T_{22}\end{array}\right]>0, U=\left[\begin{array}{cc}U_{11} & U_{12} \\ * & U_{22}\end{array}\right]>0$, diagonal matrices $Y_{1}>0, Y_{2}>0$ and matrices $M_{i}, N_{i}(i=1, \cdots, 6), \bar{M}_{j}, \bar{N}_{j}(j=1,2,3)$ with appropriate dimensions respectively, such that the following inequality holds:

$$
\Omega=\left[\begin{array}{ccccccc}
\Psi & \sigma \Gamma_{1}^{T} & \tau \Gamma_{2}^{T} & \epsilon_{1} E_{1}^{T} & D_{1} & \epsilon_{2} E_{2}^{T} & D_{2} \\
* & -\sigma S_{1} & 0 & 0 & 0 & 0 & 0 \\
* & * & -\tau S_{2} & 0 & 0 & 0 & 0 \\
* & * & * & -\epsilon_{1} I & \epsilon_{1} J & 0 & 0 \\
* & * & * & * & -\epsilon_{1} I & 0 & 0 \\
* & * & * & * & * & -\epsilon_{2} I & \epsilon_{2} J \\
* & * & * & * & * & * & -\epsilon_{2} I
\end{array}\right]<0,
$$

where

$$
\begin{aligned}
& D_{1}=\left[\begin{array}{llllllllllll}
H_{1}^{T} \bar{M}_{1}^{T} & H_{1}^{T} \bar{M}_{2}^{T} & 0 & 0 & H_{1}^{T} \bar{M}_{3}^{T} & 0 & 0 & 0 & 0 & 0 & 0 & 0
\end{array}\right]_{12 \times 1}^{T}, E_{1}=\left[\begin{array}{llllllllllll}
-Z_{1} & 0 & 0 & 0 & Z_{3} & 0 & 0 & 0 & 0 & 0 & 0 & Z_{2}
\end{array}\right]_{1 \times 12}, \\
& D_{2}=\left[\begin{array}{llllllllllll}
0 & 0 & 0 & 0 & 0 & 0 & H_{2}^{T} \bar{N}_{1}^{T} & H_{2}^{T} \bar{N}_{2}^{T} & 0 & 0 & H_{2}^{T} \bar{N}_{3}^{T} & 0
\end{array}\right]_{12 \times 1}^{T}, E_{2}=\left[\begin{array}{llllllllllll}
0 & 0 & 0 & 0 & 0 & Z_{2} & -Z_{1} & 0 & 0 & 0 & Z_{3} & 0
\end{array}\right]_{1 \times 12} \text {. }
\end{aligned}
$$

Proof. From Theorem 1 and (2), the robust asymptotic stability condition for system (5) can be described as

$$
\Psi+\sigma \Gamma_{1}^{T} S_{1}^{-1} \Gamma_{1}+\tau \Gamma_{2}^{T} S_{2}^{-1} \Gamma_{2}+D_{1} \Delta(t) E_{1}+E_{1}^{T} \Delta^{T}(t) D_{1}^{T}+D_{2} \Delta(t) E_{2}+E_{2}^{T} \Delta^{T}(t) D_{2}^{T}<0 .
$$

On the other hand, from Schur's complements lemma, we know $\Omega<0$ in (13) is equivalent to

$$
\begin{aligned}
& \Psi+\sigma \Gamma_{1}^{T} S_{1}^{-1} \Gamma_{1}+\tau \Gamma_{2}^{T} S_{2}^{-1} \Gamma_{2}+\left[\begin{array}{ll}
\sqrt{\epsilon_{1}} E_{1}^{T} & \left(\sqrt{\epsilon_{1}}\right)^{-1} D_{1}
\end{array}\right]\left[\begin{array}{cc}
I & -J \\
-J^{T} & I
\end{array}\right]^{-1}\left[\begin{array}{c}
\sqrt{\epsilon_{1}} E_{1} \\
\left(\sqrt{\epsilon_{1}}\right)^{-1} D_{1}^{T}
\end{array}\right] \\
& +\left[\begin{array}{ll}
\sqrt{\epsilon_{2}} E_{2}^{T} & \left(\sqrt{\epsilon_{2}}\right)^{-1} D_{2}
\end{array}\right]\left[\begin{array}{cc}
I & -J \\
-J^{T} & I
\end{array}\right]^{-1}\left[\begin{array}{c}
\sqrt{\epsilon_{2}} E_{2} \\
\left(\sqrt{\epsilon_{2}}\right)^{-1} D_{2}^{T}
\end{array}\right]<0 .
\end{aligned}
$$

By Schur's complements lemma, we know (14) and (15) are equivalent. Therefore, according to $\Omega<0$ in (13), we have $\dot{V}\left(x_{t}, y_{t}\right) \leq 0$. Then, by the standard Lyapunov functional theory, it concludes that the origin of the given delayed BAM neural network system (5) is robustly asymptotically stable. This completes the proof.

\section{Numerical examples}

Example 1. Consider the system (5) with the following parameters

$$
\begin{aligned}
& A=\left[\begin{array}{cc}
3 & 0 \\
0 & 2.5
\end{array}\right], W_{1}=\left[\begin{array}{cc}
0.3 & 0.25 \\
0.2 & 0.3
\end{array}\right], W_{2}=\left[\begin{array}{ll}
0.1 & 0.2 \\
0.2 & 0.1
\end{array}\right], B=\left[\begin{array}{cc}
2.2 & 0 \\
0 & 1.7
\end{array}\right], V_{1}=\left[\begin{array}{cc}
0.1 & 0.2 \\
0.2 & 0.15
\end{array}\right], \\
& V_{2}=\left[\begin{array}{cc}
0.2 & 0.1 \\
0.1 & 0.25
\end{array}\right], H_{1}=H_{2}=\left[\begin{array}{ll}
-0.1 & 0.2
\end{array}\right]^{T}, Z_{1}=Z_{2}=Z_{3}=\left[\begin{array}{ll}
0.2 & 0.5
\end{array}\right], F(t)=\sin t, \\
& \tau(t)=1.5 \cos ^{2} t, \sigma(t)=1.2 \sin ^{2} t, d=3, h=5, \\
& f_{j}(y)=\frac{1}{2}\left(\left|y_{j}+1\right|+\left|y_{j}-1\right|\right), g_{i}(x)=\frac{1}{2}\left(\left|x_{i}+1\right|+\left|x_{i}-1\right|\right), J=0.2
\end{aligned}
$$

For this example, the criteria in [7] is NOT applicable because the neutral delays are not equal to the state delays, furthermore, this system includes uncertain parameters. Therefore, we cannot conclude whether this delayed neural network is robustly asymptotically stable or not. However, by resorting to Theorem 2 and the Matlab LMI Control Toolbox, we can obtain a set of feasible solutions. Therefore, by Theorem 2, we conclude that system (5) with the above parameters is robustly asymptotically stable. The convergence dynamics of the system in Example 1 is shown in Fig. 1. 


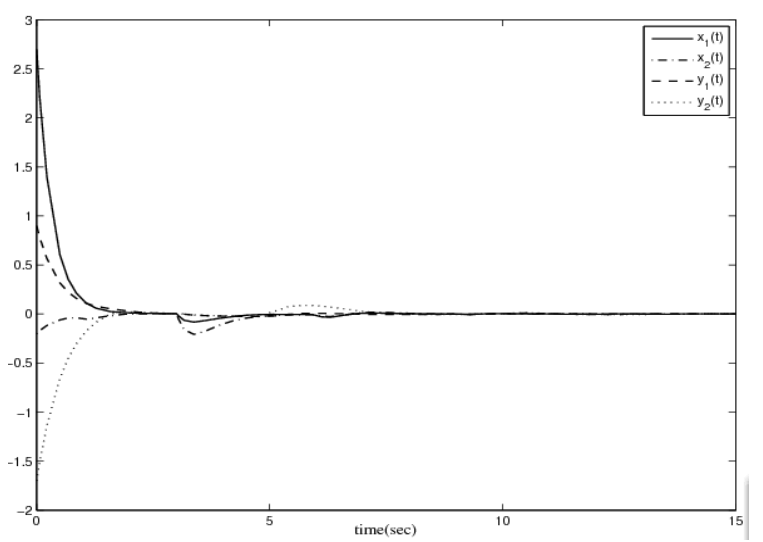

Fig.1. Convergence dynamics of the system in Example 1.

\section{Conclusion}

This paper has studied the problem of robust asymptotic stability analysis for delayed BAM neural networks of neutral-type with linear fractional uncertainties. The parameters uncertainties are expressed in a linear fractional form, which include the norm bounded uncertainties as a special case. By applying integral inequality technique together with a Lyapunov-Krasovskii functional, the novel delay-dependent stability conditions have been established. Finally, an illustrative example are given toshow the effectiveness of the obtained results.

\section{Acknowledgement}

In this paper, the research was sponsored by the National Natural Science Foundation of China (No. 61304153), the Nature Science Foundation of Tianjin (No. 15JCQNJC04200), the High School Science and Technology Development Fund Project of Tianjin (No. 20120828) and the Research Development Fund Project of Tianjin University of Technology and Education (Nos. KYQD12013, KYQD14032).

\section{References}

[1] J. Cao, Global asymptotic stability of delayed bi-directional associative memory neural networks, Appl. Math Comput. 142 (2003) 333-339.

[2] S. Arik, V. Tavsanoglu, Global asymptotic stability analysis of bidirectional associative memory neural networks with constant time delays, Neurocomputing 68 (2005) 161-176.

[3] Z. Huang, X. Luo, Q. Yang, Global asymptotic stability analysis of bidirectional associative memory neural networks with distributed delays and impulse, Chaos, Solitons, Fractals, 34 (2007) 878-885.

[4] J. Qiu, J. Zhang, J. Wang, Y. Xia, P. Shi, A new global robust stability criteria for uncertain neural networks with fast time-varying delays, Chaos, Solitons, Fract. 37 (2008) 360-368.

[5] O.M. Kwona, J.H. Park, Delay-dependent stability for uncertain cellular neural networks with discrete and distribute time-varying delays, J. Franklin Institute 345 (2008) 766-778.

[6] Ju H. Park, C.H. Park, O.M. Kwon, S.M. Lee, A new stability criterion for bidirecrtional associative memory neural networks of neutral-type, Appl. Math. Comput. 199 (2008) 716-722.

[7] J. Liu, G. Zong, New delay-dependent asymptotic stability conditions concerning BAM neural networks of neutral type Neurocomputing 72 (2009) 2549-2555. 